\title{
Identification of lipases with activity towards monoacylglycerol by criterion of conserved cap architectures
}

\author{
Lina Riegler-Berket ${ }^{1,2}$, Andrea Leitmeier ${ }^{1,2}$, Philipp Aschauer ${ }^{1,2}$, Ingrid Dreveny ${ }^{3}$ and Monika \\ Oberer $^{1,2^{*}}$
}

1) Institute of Molecular Biosciences, University of Graz; Austria

2) BioTechMed-Graz, Austria

3) School of Pharmacy, University of Nottingham, University Park, Nottingham, NG7 2RD, UK

* to whom correspondence should be addressed

\begin{abstract}
:
Monoacylglycerol lipases (MGL) are a subclass of lipases that predominantly hydrolyze monoacylglycerol into glycerol and free fatty acid. MGLs are ubiquitous enzymes across species and play a role in lipid metabolism, affecting energy homeostasis and signaling processes. Structurally, MGLs belong to the $\alpha / \beta$ hydrolase fold family with a cap covering the substrate binding pocket. Analysis of the known 3D structures of human, yeast and bacterial MGLs revealed striking similarity of the cap architecture. Since MGLs from different organisms share very low sequence similarity, it is difficult to identify MGLs based on the amino acid sequence alone. Here, we investigated whether the cap architecture could be a characteristic feature of this subclass of lipases with activity towards MG and whether it is possible to identify MGLs based on the cap shape. Through database searches, we identified the structures of five different candidate $\alpha / \beta$ hydrolase fold proteins with unknown or reported esterase activity. These proteins exhibit cap architecture similarities to known human, yeast and bacterial MGL structures. Out of these candidates we confirmed MGL activity for the protein LipS, which displayed the highest structural similarity to known MGLs. Two further enzymes, Avi_0199 and VC1974, displayed low level MGL activities. These findings corroborate our hypothesis that this conserved cap architecture can be used as criterion to identify lipases with activity towards MGs.
\end{abstract}

Keywords: Monoacylglycerol lipase, monoglyceride lipase, lipase cap, conserved cap architecture, lipase lid, enzyme kinetics

\section{Introduction}

Monoacylglycerol lipases (MGLs) are hydrolytic enzymes found in all domains of life. As their physiological role, MGLs metabolize lipid intermediates involved in energy homeostasis by producing fatty acids for energy production and in signaling processes (e.g. endocannabinoid signaling) [1-3]. MGLs from human [4-6], rat [7], yeast [8], plant [9] and several bacterial [2,10-12] species have been studied biochemically since the 1960s.

\section{Abbreviations}

MGL, monoacylglycerol lipase; $A B H, \alpha / \beta$ hydrolase; $M G$, monoacylglycerol; $h M G L$, monoacylglycerol lipase from Homo sapiens; bMGL, monoacylglycerol lipase from Bacillus species. H-257 
MGLs are a subclass of lipases that belong to the $\alpha / \beta$ hydrolase $(A B H)$ fold family, one of the largest groups of structurally related enzymes with a broad range of catalytic functions. These 'classical' MGLS (EC 3.1.1.23) hydrolyze glycerol monoesters of long-chain fatty acids (monoacylglycerols, MGs) into glycerol and the corresponding fatty acid. The reaction is carried out by residues of a catalytic triad with the nucleophilic serine embedded in a GXSXG consensus sequence. MGLs have a narrow substrate range; little, if any, hydrolytic activity is observed on long chain di- and triacylglycerols, cholesterol esters or more complex lipid substrates. Short chain esters, methyl or ethyl esters are sometimes hydrolyzed. This narrow substrate range distinguishes MGLs from numerous other lipases with a relatively broad substrate range that may also include activity towards MGs. These lipases often display activity on a similar level towards other, more complex substrates (e.g. tri- or diacylglycerols, phospholipids), as for example observed for pancreatic lipase related protein 2 or bile salt stimulated lipase [13-15]. Based on the substrate preferences, theses lipases are classified into different subclasses of hydrolases e.g. EC 3.1.1.3 triacylgylcerol lipase, EC 3.1.1.4 phospholipase A(2); EC 3.1.1.5 lysophospholipase.

The first 3D structures of any of these 'classical' MGLs have been published relatively recently including those of Homo sapiens (hMGL, UniProt-ID Q99685) [16-19], Bacillus species. H-257 (bMGL, UniProt-ID P82597)[20,21], and Saccharomyces cerevisiae (YJU3p, UniProt-ID P28321) [22]. These structures confirmed that MGLs harbor the ABH fold characterized by a twisted central $\beta$-sheet flanked on both sides by $\alpha$-helices. The conserved catalytic triad is comprised of a nucleophile after $\beta 5$, an acidic residue after strand $\beta 7$ and a histidine in the loop after the last strand. Variations to the canonical ABH fold are common. In lipases, so-called cap or lid regions are often inserted and are involved in regulating the accessibility to the active site and are highly diverse in size, shape and topology [23-25]. In the three MGL orthologues with known 3D structures, the cap is inserted after strand $\beta 6$ and comprises 56, 40 and 59 amino acids in hMGL, bMGL and YJU3p, respectively. We noted that these residues form a cap structure with remarkably high similarity in the overall architecture despite harboring different secondary structure elements, sequence lengths and less than $20 \%$ sequence identity $[20,22]$. This unexpected resemblance of the cap architectures in MGL orthologues could not have been predicted by sequence analysis and stirred our interest for this study. We hypothesized that this cap architecture could be a hallmark of classical MGLs. If correct, it should be possible to infer activity towards MG substrates solely based on the three-dimensional shape of the cap structure of ABH fold enzymes containing the GXSXG lipase motif.

To test this hypothesis, we searched the Protein Data Bank (PDB Europe) for structures belonging to the $\mathrm{ABH}$ fold family that harbor a similar cap architecture and tested these for MGL activity. We selected five proteins, Avi_0199 (a putative hydrolase), CesA [26], LipS [27], TtEst [28] and Vc1974, and 
tested these for esterase and MGL activity; the latter has not previously been reported for any of these proteins. For two of these proteins (Vc1974 and Avi_0199) this study represents the first biochemical characterization of their activity. In support of our hypothesis and based on our structural search, we reveal that LipS, a previously reported thermophilic lipase from Symbiobacterium thermophilum [27], has significant activity towards MG and can be described as MGL.

\section{Material and methods}

\subsection{Identification and analysis of potential monoacylglycerol lipases}

To identify potential MGLs based on the cap architecture as defining criterion we used the protein structure comparison service PDBeFold at the European Bioinformatics Institute (http://www.ebi.ac.uk/msd-srv/ssm) [29]. The search was performed as pairwise 3D alignments of the full-length MGL structures with PDB codes 3PE6 and 3RM3 against the whole PDB archive using default search parameters. The results were manually evaluated by visualization and structure superposition. Additionally, we searched the literature for newly determined 3D structures with similar features. The PyMOL Graphics System was used for visualization and analysis of protein structures [30]. Structurebased alignments of separated core and cap structures were performed with TM-align [31]. Proteins that fulfilled the cap domain architecture criterion (a cap insertion after beta strand $\beta 6$ with a shape resembling that of the described MGLs and that is covering the catalytic cavity) with ABH fold core domain, a conserved GXSXG motif [23] and an intact catalytic triad were chosen to be tested for MGL activity.

\subsection{Cloning, expression and purification}

The genes for the proteins Avi_0199, Vc1974 and CesA were purchased from the DNASU Plasmid Repository (Arizona State University, USA). Avi_0199 and CesA in bacterial expression vector pSpeedET were used directly for protein expression and purification. Vc1974 was amplified by PCR with the forward primer 5'-CAGCACGGATCCATGCTCTCTAACC-3' and reverse primer 5'-GTCCAGCTCGAGCAAATCGATTATTGAGTGG-3' and cloned using BamHI and Xhol restriction sites into the pProEx-HTb expression vector (Thermo Fisher Scientific, Waltham, MA, USA). The gene for TtEst was purchased codon-optimized for E.coli expression from Eurofins (Vienna, Austria) and subcloned into pST50trc1 [32] with the Gibson Assembly Cloning Kit from New England BioLabs (Frankfurt am Main, Germany). The vector was cut with restriction enzymes Ndel and NgolV and primers forward 5'CTTTAAGAAGGAGATATACATATGGCACAGCGTGTGAAGATTAC-3' and reverse 5'AGTGGTGGTGGTGGTGGTGGCCTTCCGTGTGCACTTTGCG-3' were used for amplification of the gene. The gene for LipS in PET21a was kindly provided by Prof. Dr. Wolfgang Streit from the University of Hamburg [27]. The bMGL expression construct was reported previously [20]. 
For protein expression of Avi_0199, Vc1974 and CesA the plasmids were transformed into BL21(DE3) codon plus cells (Stratagene Agilent Technologies, Santa Clara, USA). The main cultures were inoculated in a 1:100 ratio with overnight culture in $\mathrm{LB}$ medium and grown at $37^{\circ} \mathrm{C}$ until an $\mathrm{OD}_{600}$ of 0.4-0.6. Expression was induced upon addition of $1 \mathrm{mM}$ IPTG for approximately. $20 \mathrm{~h}$ at $25^{\circ} \mathrm{C}$ for Vc1974, and $4 \mathrm{~h}$ at $37^{\circ} \mathrm{C}$ for Avi_0199 and CesA. Cells were harvested by centrifugation for $30 \mathrm{~min}$ at $4^{\circ} \mathrm{C}$ and $2,160 \mathrm{~g}$, the cell pellet was re-suspended in lysis buffer (50 mM HEPES or $50 \mathrm{mM}$ sodium phosphate buffer pH 8.0, $300 \mathrm{mM} \mathrm{NaCl}, 1 \mathrm{mM}$ TCEP, $20 \mathrm{mM}$ imidazole) and lysed by sonication on ice. Cell lysates were clarified by centrifugation for $30 \mathrm{~min}$ at $4^{\circ} \mathrm{C}$ and $23,600 \mathrm{~g}$. The proteins were purified using a $5 \mathrm{ml} \mathrm{His-Trap} \mathrm{FF} \mathrm{crude} \mathrm{column} \mathrm{(GE} \mathrm{Healthcare,} \mathrm{Little} \mathrm{Chalfond,} \mathrm{UK)} \mathrm{by} \mathrm{gradient} \mathrm{elution} \mathrm{from} 20$ to $300 \mathrm{mM}$ imidazole. Vc1974 was dialyzed against $50 \mathrm{mM}$ sodium phosphate buffer $\mathrm{pH}$ 8.0. The Histags of Avi_0199 and CesA were cleaved during dialysis with TEV-protease. The TEV protease and cleaved His-tag were removed by reverse affinity chromatography before dialyzing against $50 \mathrm{mM}$ $\begin{array}{lllll}\text { sodium } & \text { phosphate } & \text { buffer } & \text { 8.0. }\end{array}$ bMGL, LipS and TtEst were expressed and purified by immobilized metal affinity chromatography as described previously $[20,27,28]$. The purity of all proteins was verified by SDS-PAGE. Christoph Heier (University of Graz) kindly provided hormone sensitive lipase (HSL) from mouse expressed in Cos7 cells.

\subsection{Activity Assay}

Assays for bMGL, LipS, TtEst, Avi_0199, Vc1974 and CesA were performed at $37^{\circ} \mathrm{C}$, whereas assays with LipS were performed at $70^{\circ} \mathrm{C}$ due to the reported highest activity towards $p$-nitrophenyl ( $p N P$ ) dodecanoate at $70^{\circ} \mathrm{C}[27]$.

\subsubsection{Esterase activity assay}

Esterase activity was studied by incubation of enzymes (0.01-10 $\mu \mathrm{M})$ with $1 \mathrm{mM} p \mathrm{NP}$-acyl ester substrate in $100 \mu$ of assay buffer ( $0.1 \mathrm{M}$ sodium phosphate buffer $\mathrm{pH} 8.0$ ) for $10 \mathrm{~min}$ followed by reading the absorbance against a blank at $405 \mathrm{~nm}$. For quantification of $p$-nitrophenol, the exctinction coefficient of $14,220 \mathrm{M}^{-1} \mathrm{~cm}^{-1}$ was used. Stock solutions of the short chain substrates $p N P$ acetate ( $p N P$ C2), -butyrate ( $p N P-C 4: 0)$ and -octanoate ( $p N P-C 8: 0)$ were dissolved in ethanol and directly used for the assays, whereas medium and long chain substrates $p$-NP decanoate ( $p$ NP-C10:0), -dodecanoate ( $p$ NP-C12:0) and -palmitate ( $p$ NP-C16:0) dissolved in chloroform were dried under nitrogen, sonicated with assay buffer and finally centrifuged for $5 \mathrm{~min}$ at 20,820 g. The reaction was stopped by adding $100 \mu \mathrm{l}$ chloroform/methanol/heptane (9:10:7) and after centrifugation for $5 \mathrm{~min}$ at 20,820 $\mathrm{g}$ the absorbance of the upper phase at $405 \mathrm{~nm}$ was measured using a NanoDrop ${ }^{\circledR} \mathrm{ND}-1000$ spectrometer. Assays were performed in triplicates in at least three independent experiments. 


\subsubsection{MG hydrolase activity assay}

The MG hydrolase assays were essentially performed as previously described [8,21]. $10 \mu \mathrm{l}$ of 50 $100 \mathrm{nM}$ of LipS or bMGL and 10-50 $\mu$ M of Avi_0199, CesA or Vc1974 were incubated with $100 \mu \mathrm{l} 1 \mathrm{mM}$ substrate for $20 \mathrm{~min}$. Substrates used were purchased from Sigma-Aldrich and had different chain lengths and saturation: 1-lauroyl-rac-glycerol (MG-C12:0), 1-myristoyl-rac-glycerol (MG-C14:0), 1monopalmitoleoyl-rac-glycerol (MG-C16:1), 1-stearoyl-rac-glycerol (MG-C18:0) and 1-oleoyl-racglycerol (MG-C18:1). Kinetic studies were performed with $3 \mathrm{nM}$ protein and $0.03 \mathrm{mM}-2 \mathrm{mM}$ concentration of substrate for calculation of the kinetic parameters $V_{\max }, K_{M}, k_{c a t}$. Reactions were stopped after $15 \mathrm{~min}$, which is in the linear range of the reaction (Fig. 4A, 4B). The data were analyzed with GraphPad Prism 5.0 (GraphPad Software, California, USA). All assays were performed in triplicates and the graphs are representative for at least three independent biological replicates.

\subsubsection{Diacylglycerol hydrolase activity assay}

Diacylglycerol hydrolase activity was tested for LipS and Avi_0199. The substrate was prepared by emulsifying dioleoylglycerol (mixture of 1,3- and 1,2-isomers) and phosphatidylcholine in assay buffer by sonication, with subsequent adjustment to $5 \%$ BSA to reach a final concentration of $450 \mu \mathrm{M}$ dioleoylglycerol in $54 \mu \mathrm{M}$ phosphatidylcholine. $50 \mu \mathrm{g}$ enzyme in $100 \mu \mathrm{l}$ assay buffer was added to 100 $\mu \mathrm{l}$ substrate and the mixture was incubated for $1 \mathrm{~h}$ at $37^{\circ} \mathrm{C}$ and free fatty acids were measured with the NEFA kit (Wako Chemicals GmbH, Germany) according to the manufacturer's instructions. The activity of HSL was tested in Cos-7 lysates. The experiments were performed in duplicates and are representative for at least three independent biological replicates.

\section{Results}

\subsection{Identification of MGL-like proteins based on their 3D structure}

Our observation that MGLs across the three kingdoms of life possess an overall similar cap architecture prompted us to investigate our hypothesis that it may be possible to identify enzymes with MGL activity based on this structural characteristic. To this end, PDB database searches were conducted. A PDBeFold structure similarity search using the whole structure of hMGL, PDB code 3PE6 [19], as query resulted in 193 hits. Out of these, 28 hits corresponded to chains of bMGL, YJU3p or hMGL structures. Using the 3D structure of bMGL deposited under accession code 3RM3 [20] as a search query, the hit list contained 59 structures, among these 25 were chains of bMGL and YJU3p structures including structures in different crystal forms and ligand complexes. A list containing the hits obtained is included as Supplementary Table. From the remaining hits, redundant entries for any given protein were removed. The hit list from the PDBe search contained proteins harboring ABH-domains with caps of similar or of distinct shapes compared to the MGL-cap, and these either contained or lacked the 
consensus GXSXG lipase motif. This left 55 candidate protein structures from the first search and 12 proteins from the second search, all of whom comprised an $\mathrm{ABH}$ fold core. The hit list contains a vast array of enzymes described e.g. as carboxyl esterases, bromoperoxidases and multiple biochemically uncharacterized protein structures from structural genomics consortia. These remaining candidates were then subjected to the following selection criteria: i) an intact catalytic triad composed of a serine nucleophile embedded in the conserved lipase GXSXG motif, an acidic residue and a histidine; and ii) the presence of a cap structure. The cap structures of these proteins were then further examined and compared to structurally characterized MGLs. Cap residues in known MGLs were identified as depicted in Fig. 1, comprising Leu161-Leu216 in hMGL, Asp124-Pro163 in bMGL and Ile153-Ser211 in YJU3p. These cap structures start after $\beta$-sheet $\beta 6$ with the cap-helix $\alpha 1$ covering the "upper" part of the binding pocket (Fig. 2A). In case of YJU3p, the first helix corresponding to $\alpha 1$ is interrupted by a small bend. The following amino acids then cross the substrate binding pocket diagonally; hMGL and YJU3p display an additional short helix (termed $\alpha 2$ in Fig. 2A) in this stretch. The third cap helix ( $\alpha 3$ ) is located almost parallel to $\alpha 1$ followed by a second loop region, which brings the amino acids closer towards $\alpha 1$ and restricts access to the binding pocket on the other side of the protein. In bMGL, a small $\beta$-sheet is formed between the diagonal stretch and the last loop region (Tyr141-Asp143 $\left(\beta_{1}\right)$, Lys161-Pro163 $\left.\left(\beta_{2}\right)\right)$. Despite differences in lengths and secondary structure elements, this overall cap architecture adopts a shape that is conserved between MGL orthologues [20,22] (Fig. 2). 

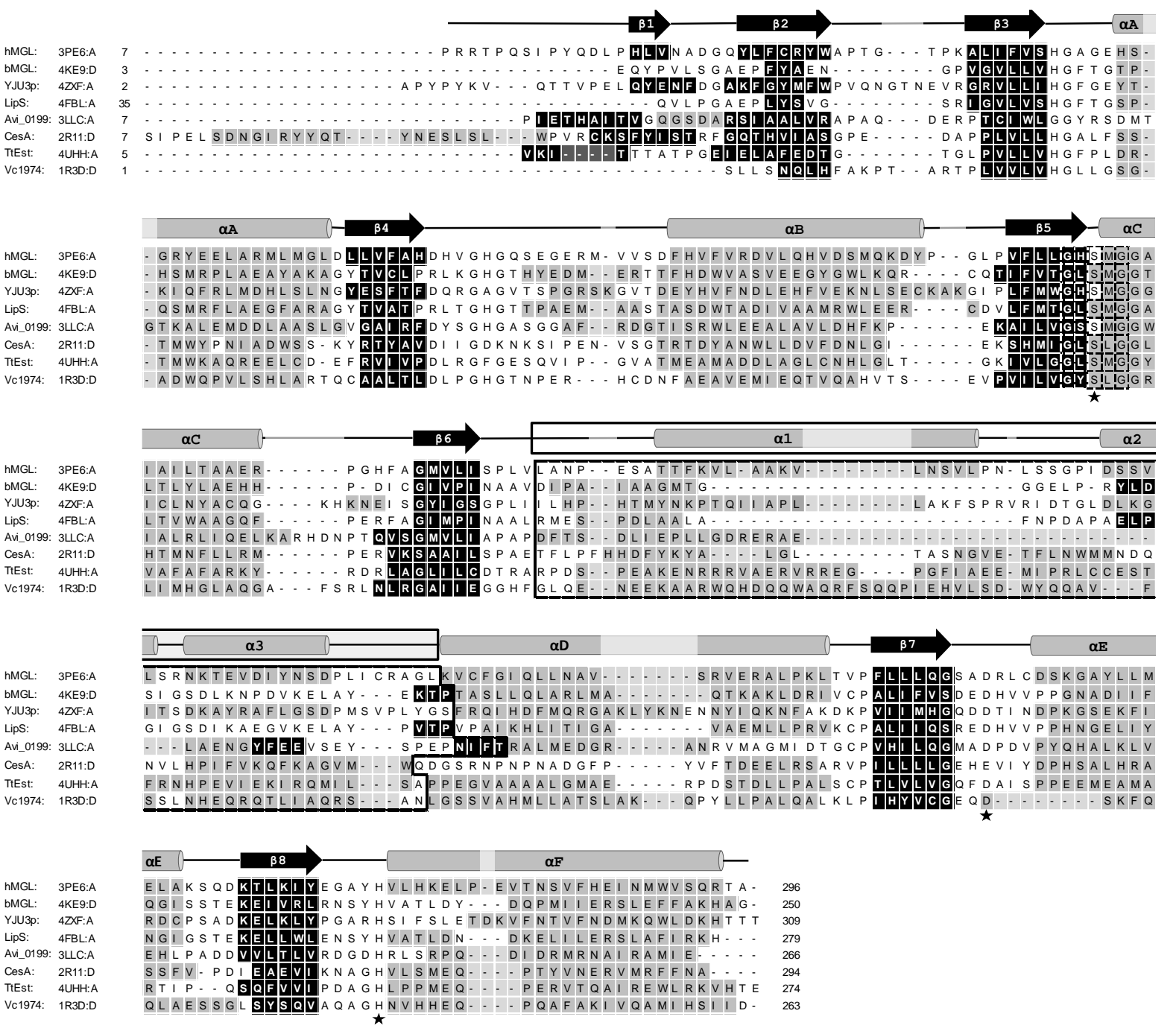

Figure 1. Structure-based multiple sequence alignment of MGLs with known 3D structures and proteins identified in the cap-similarity search. Sequences are labelled by the protein name followed by the PDB code and respective chain used in the alignment. Residues highlighted in white on black background adopt $\beta$-strand conformation, whereas residues in black letters on grey background adopt $\alpha$-helical conformation. Secondary structure elements of hMGL (PDB code 3PE6) are labelled according to the nomenclature used for $A B H$ fold proteins and are depicted above the sequence. The $\alpha / \beta$ hydrolase core is formed by $\beta$-strands $\beta 1-\beta 8$ (bMGL, LipS and $\mathrm{V} c 1974$ do not have $\beta 1$ ) and six $\alpha$-helices $\alpha A-\alpha F$. The cap is located in an insertion between $\beta 6$ and $\alpha D$ and is highlighted by a black frame. Note the differences in the secondary structure elements of the caps in these structures. The catalytic residues are marked with an asterisk and the conserved GXSXG motif is underlined with a dashed line. The structure based multiple sequence alignment was performed with PROMALS3D [33].

We then examined the similarities between the cap structures from candidate proteins and known MGLs in terms of the topology, size and architecture by manual inspection and TM alignment-scores. Subsequently, a selection of proteins that fulfilled the criteria and had similar cap architectures were subjected to experimental testing.

The selected structures included those of proteins Vc1974 (PDB code 1R3D, New York Structural Genomics Research Consortium, unpublished, UniProt-ID Q9KQM4), CesA, (PDB code 2R11, Joint 
Center for Structural Genomics (JCSG), unpublished UniProt-ID P96688), Avi_0199, (PDB code 3LLC, JCSG, unpublished, UniProt-ID B9JYM4) and LipS (PDB code 4FBL [27]) (Table 1 and Figure 2). Other proteins displayed more divergence in the cap or did not harbor the conserved GXSXG lipase consensus motif. The structures of Vc1974 and CesA were only found with hMGL as a search query and the structure of Avi_0199 was detected using bMGL as query. Notably, the structure of LipS showed the highest Z-score in both hit lists. We also investigated a fifth protein as potential MGL, TtEst (PDB code 4UHH [28]), which we identified through a regular literature survey. Results from the pairwise comparisons and 3D alignments of the structures selected through the cap structure similarity search and literature survey with PDBeFold are listed in Table 1.

PDB-ID;

PDBeFold result

\begin{tabular}{|c|c|c|c|c|c|c|c|c|c|}
\hline \multirow[b]{2}{*}{$\begin{array}{c}\text { Protein- } \\
\text { name }\end{array}$} & \multirow[b]{2}{*}{$\begin{array}{c}\text { Resolution } \\
\text { (Å) }\end{array}$} & \multirow[b]{2}{*}{ Organism } & \multirow[b]{2}{*}{$\begin{array}{c}\text { Accession } \\
\text { number }\end{array}$} & \\
\hline & & & & Query & Z-score & $\begin{array}{c}\text { RMSD } \\
\text { (Å) }\end{array}$ & $\mathbf{N}_{\text {algn }}$ & $\mathrm{N}_{\text {res }}$ & $\%$ seq \\
\hline \multirow{2}{*}{ LipS } & \multirow{2}{*}{ 4FBL; 1.99} & Symbiobacterium & \multirow{2}{*}{ BAD41030.1 } & $3 \mathrm{RM3}$ & 18.81 & 0.80 & 234 & 246 & 52.6 \\
\hline & & thermophilum IAM 14863 & & 3PE6 & 12.61 & 1.87 & 229 & 246 & 19.7 \\
\hline CesA & 2R11; 1.96 & B.subtilis 168 & P96688.1 & 3PE6 & 10.21 & 2.07 & 201 & 287 & 16.9 \\
\hline TtEst & $4 \mathrm{UHH} ; 1.06$ & Thermogutta terrifontis & KR002593 & 3PE6 & 9.29 & 2.26 & 205 & 273 & 17.6 \\
\hline \multirow{2}{*}{ Avi_0199 } & \multirow{2}{*}{$3 \mathrm{LLC} ; 1.80$} & \multirow{2}{*}{ Agrobacterium vitis $\mathrm{S} 4$} & WP_0126546 & $3 \mathrm{RM} 3$ & 9.22 & 2.13 & 200 & 261 & 17.5 \\
\hline & & & 50 & 3PE6 & 10.29 & 2.08 & 210 & 261 & 19.5 \\
\hline Vc1974 & 1R3D; 1.90 & $\begin{array}{l}\text { Vibrio Cholerae } 01 \text { biovar } \\
\text { El Tor str. N16961 }\end{array}$ & NP_231608 & 3PE6 & 7.69 & 2.55 & 187 & 257 & 19.3 \\
\hline
\end{tabular}

Table 1: List of selected potential MGLs as identified via comparison of the cap-architectures. PDBeFold results from 3D alignment of query structures hMGL (PDB code 3PE6) and bMGL (PDB code 3RM3) with the selected proteins.

\subsection{Evaluation of MGL features in the selected candidate structures}

In order to evaluate whether the identified proteins exhibit MGL activity we analyzed their overall structures, active sites and searched the literature for any available data that would support this. The structures of Avi_0199 and Vc1974 were deposited as putative hydrolases by the JCSG and NYSGRC, respectively, but have not been published. Based on structural and sequence alignments to $\mathrm{hMGL}$, we identified the catalytic triad for Avi_0199 as comprising of Ser113, Asp216 and His246, and for Vc1974 as Ser91, Asp218 and His240 (Fig. 1, 2). CesA is a hydrolase from the B. subtilis strain 168 and has previously been biochemically characterized [26]. The structure was solved by the JCSG in 2007 but is as yet unpublished. The protein has reported activity against different esters with the highest activity reported for rac-ibuprofen methyl ester $\left(25.4 \mu \mathrm{mol} \cdot \mathrm{min}^{-1} \cdot \mathrm{mg}^{-1}\right)$ [26]. CesA shares $98 \%$ sequence identity with a naproxen esterase from $B$. subtilis, Thai 1-8 [34]. Based on the high sequence conservation with the naproxen esterase and sequence alignment with $\mathrm{hMGL}$, the catalytic triad of 
CesA is defined as Ser130, Glu245 and His274 (Fig. 1). LipS is a thermostable lipase derived from a metagenomics library and shows $100 \%$ amino acid sequence identity to a lipase from Symbiobacterium thermophilum. It was characterized as a thermostable lipase with a temperature optimum at $70^{\circ} \mathrm{C}$. LipS was tested with a wide range of substrates, yet no MGs, even though the structural similarity to hMGL is discussed [27]. The highest activity was measured against $p$ NP-acyl esters, in particular pNP-C10 $\left(12.0 \mu \mathrm{mol} \cdot \mathrm{min}^{-1} \cdot \mathrm{mg}^{-1}\right)$. The catalytic triad is composed of Ser126, Asp227, and His257 [27]. The thermostable esterase TtEst from Thermogutta terrifontis exhibits hydrolase activity against $p$ NP-C2 to $p N P-C 5$ substrates with the highest activity against $p N P-C 3\left(1.07 \mu \mathrm{mol} \cdot \mathrm{min}^{-1} \cdot \mathrm{mg}^{-1}\right)$. The catalytic activity is mediated by residues Ser101, Asp222 and His250 [28].

All identified potential MGLs share low sequence identity with human MGL (Table 1). The amino acid sequences of the candidate proteins can be aligned based on their structural elements and clearly show the conserved ABH core topology (Fig. 1 and 2B). Notably, the sequence and structural variations in the cap region are more pronounced than in the core domain (Fig. 1,2). However, the 3D structures of the cap of the five selected proteins overall show a Z-like shape as observed in MGLs (Fig. 2A) encompassing the following residues: Arg154-Pro194 in LipS; Asp147-Thr185 in Avi_0199; Thr158Trp207 in CesA; Arg129-Ala185 in TtEst and Gly122-Asn179 in Vc1974. LipS highly resembles bMGL, in terms of sequence length, structure and secondary structure elements of the cap. In LipS, 41 residues form the cap (Fig. 2A, red) with one $\alpha$-helix ( $\alpha 1$ ) superimposing onto $\alpha 1$ in hMGL. The arrangement of a small $\beta$-sheet formed from residues of the diagonal stretch (Glu172-Pro174, $\beta_{1}$ ) and residues of the second loop in the cap (Val192-Pro194, $\beta_{2}$ ), is highly similar to the bMGL cap structure (Fig. 2A, orange). Avi_0199 harbors the shortest cap region with 39 amino acids (Fig. 2A, blue). The first $\alpha$-helix ( $\alpha 1$ ) of the cap and the following $\beta$-sheet superpose well with helix $\alpha 1$ and the following diagonal loop region of hMGL (Fig. 2A, black). However, the diagonal stretch in Avi_0199 is much shorter (by eight residues) compared to hMGL and secondary structure elements comparable to helices $\alpha 2$ and $\alpha 3$ are missing. Thus, the cap still forms a Z-shape, but of overall smaller dimensions. The cap of Vc1974 has a length of 58 amino acids that form three helices $(\alpha 1, \alpha 2, \alpha 3)$ connected by short linkers (Fig. 2A, cyan). Compared to the hMGL cap, the first $\alpha$-helix is longer (by 10 residues), and a shorter diagonal stretch is formed entirely by the second helix. Following a short loop, a third helix is angled back towards the end of $\alpha 1$ similar to the last loop in the hMGL cap structure. However, helices $\alpha 2$ and $\alpha 3$ of hMGL do not align well with $\alpha 2$ and $\alpha 3$ of Vc1974. The cap of TtEst (57 amino acids, Fig. 2A, purple) shares $\alpha$ helical elements in all sections of the Z-shape. Compared to the helix $\alpha 1$ in $\mathrm{hMGL}$, the first cap helix $\alpha 1$ of TtEst is tilted by approx. $45^{\circ}$, whereas $\alpha 2, \alpha 3$ and $\alpha 4$ align well with the other hMGL cap structural elements. The cap of CesA (49 residues, Fig. 2A, pink) comprises three helices in an overall shortened Z-shape. Similar to Vc1974, helix $\alpha 2$ forms a short diagonal stretch followed by a loop region that runs 
parallel to $\alpha 3$ in hMGL. However, this long loop positions the last helix ( $\alpha 3)$ in the direction back towards $\alpha 1$. Similar to Vc1974, CesA and hMGL $\alpha 1$ helices align well, whereas $\alpha 2$ and $\alpha 3$ align poorly. 
A

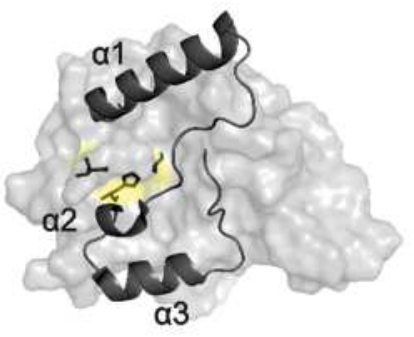

hMGL

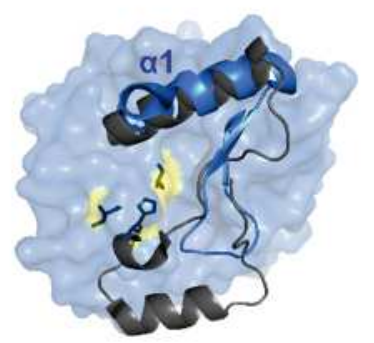

Avi_0199

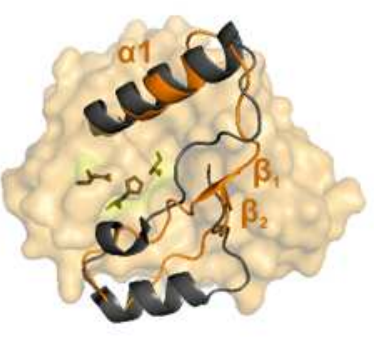

bMGL

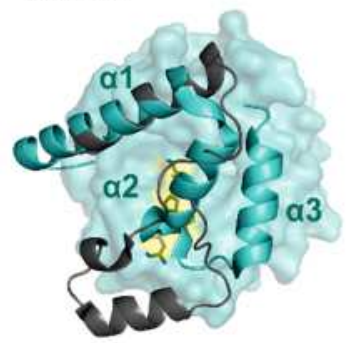

Vc1974
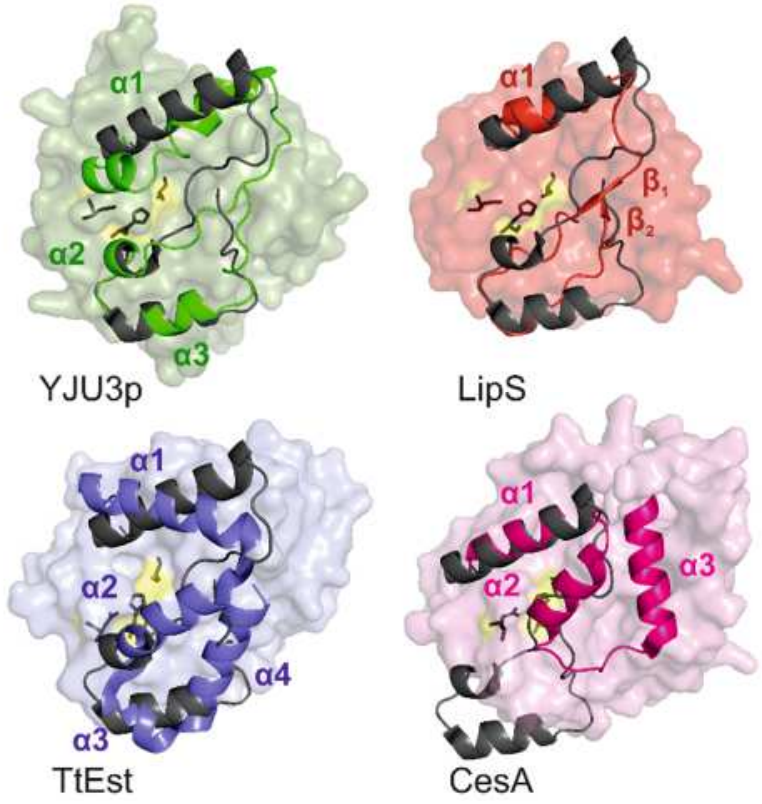

B
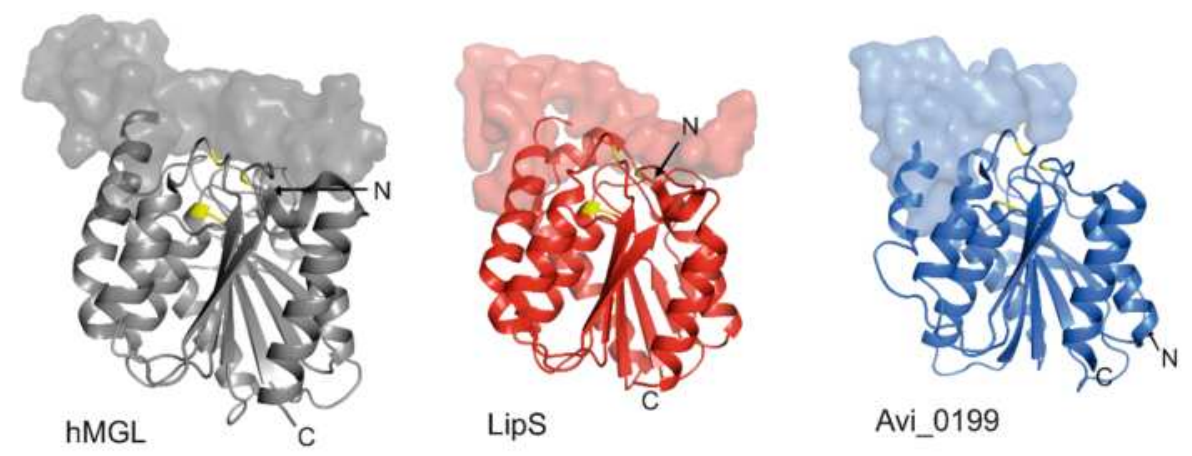

Avi_0199
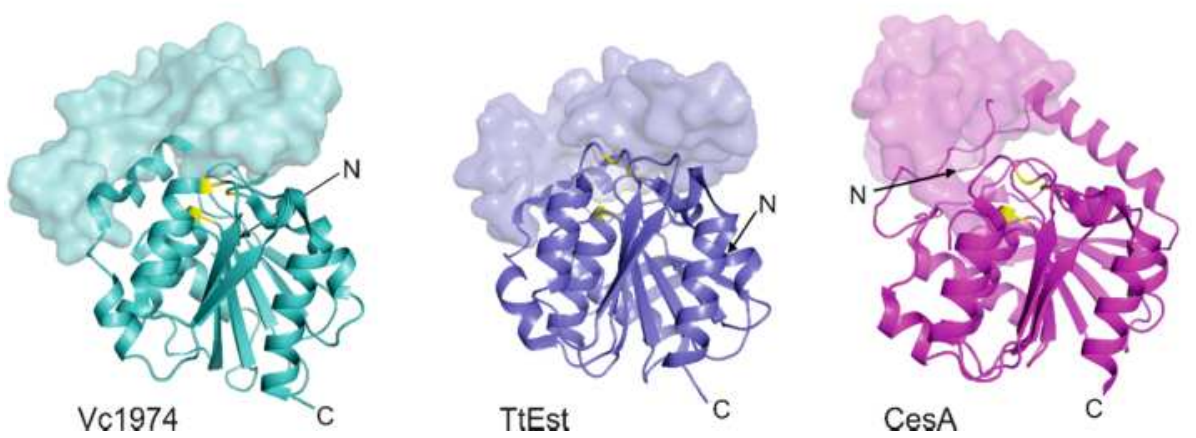

Figure 2 Structures of known MGLs and candidate MGLs compared to the hMGL structures. Color coding is as follows: characterized MGLs: bMGL (PDB code 4KE9, orange), YJU3p (PDB code 4ZXF, green) and hMGL (PDB code 3PE6, grey); candidate MGLs: LipS (PDB code 4FBL, red), Avi_0199 (PDB code 3LLC, blue), Vc1974 (PDB code 1R3D, cyan), TtEst (PDB code 4UHH, purple), CesA (PDB code 2R11, pink). A) Cap structures of MGLs and candidate MGL proteins aligned to the hMGL cap. For clarity, proteins are shown in the same orientation. The cores of the respective proteins are shown in surface representation whereas the caps are in cartoon representation. The residues of the catalytic triad are shown in black stick representation and highlighted yellow on the surface. Selected secondary structure elements are labelled. B) ABH core domains from identified proteins with similar cap architecture as hMGL. All structures are shown in the same orientation with the catalytic cores 
displayed in cartoon representation while the cap regions are shown in surface representation for clarity. The positions of catalytic triad residues are colored yellow. $\mathrm{N}$ - and $\mathrm{C}$-termini are labelled. All proteins adopt the typical ABH fold, a twisted $\beta$-sheet in the center, which is flanked by $\alpha$-helices on both sides.

\subsection{Search for a signature definition of MGL cap structures}

Further analysis of the alignments of known MGL structures with the identified candidate proteins was performed using TM-align [31]. Since the RMSD is strongly dependent on the size of the proteins and small local structural deviations can lead to high RMSD, we used the TM-score for a qualitative description of the similarity of the overall topology of the structures. The TM-score is more sensitive to the global topology, because it is normalized and not dependent on the protein size. Statistically a TM-score below 0.3 means random structural similarity and values between 0.5-1.0 indicate the same fold of the structures [31].

For hMGL, bMGL and YJU3p, crystal structures with different cap conformations ranging from open to closed are observed independently of ligand binding [16-19,21,22]. Here, we used the closed structures for comparison to the selected proteins, yet the overall shape of the cap remains independent of the conformation. In hMGL and YJU3p, the conformational changes occur predominantly in the $\alpha 1$ helix, whereas in bMGL Ile145 in the diagonal stretch of the cap acts as gatekeeper of the substrate binding pocket [21]. When comparing the open and closed conformation of human MGL (PDB codes 3HJU and 3PE6, respectively) the TM-score is 0.590, which is well above the 'same-fold' threshold.

The cap and core regions from candidate proteins were analyzed separately and compared to hMGL. As expected, all core regions reach a TM-score higher than 0.5 (0.689-0.818) (Table 2). The structural variance between the cap regions of MGLs and the 5 candidate structures is reflected in their lower TM-scores $(<0.5)$ compared to the core region alignment scores (Table 2). The alignments of the known MGL caps, YJU3p and bMGL with hMGL showed score values of 0.482 and 0.442 , respectively. The alignment with the LipS cap resulted in the lowest C $\alpha$ backbone RMSD ( $2.40 \AA$ over 40 residues) and a TM-score of 0.430 , which is close to the values observed for known MGLs. The cap alignments of Vc1974, TtEst, CesA and Avi_0199 show TM-scores of 0.374, 0.373, 0.312 and 0.279, respectively. 


\begin{tabular}{|c|c|c|c|c|c|c|}
\hline & \multirow[b]{2}{*}{ PDB code } & \multicolumn{2}{|r|}{ Cap } & \multicolumn{3}{|c|}{ Core } \\
\hline & & TM-score & $\operatorname{RMSD}(\AA)$ & TM-score & RMSD & (Å) \\
\hline YJU3p & $4 Z X F$ & 0.482 & $2.88 \quad(50 / 59)$ & 0.856 & 1.96 & $(219 / 249)$ \\
\hline bMGL & 4KE9 & 0.442 & $2.52 \quad(39 / 40)$ & 0.796 & 2.46 & $(207 / 233)$ \\
\hline LipS & $4 \mathrm{FBL}$ & 0.430 & $2.40 \quad(40 / 41)$ & 0.784 & 2.16 & $(202 / 233)$ \\
\hline Vc1974 & 1R3D & 0.374 & $3.42 \quad(35 / 58)$ & 0.689 & 2.83 & $(190 / 233)$ \\
\hline TtEst & $4 U \mathrm{HH}$ & 0.373 & $3.72 \quad(48 / 57)$ & 0.818 & 2.61 & $(207 / 233)$ \\
\hline CesA & 2R11 & 0.312 & $3.17 \quad(29 / 49)$ & 0.766 & 3.09 & $(211 / 238)$ \\
\hline Avi_0199 & $3 L L C$ & 0.279 & (30/39) & 0.794 & 2.40 & $(208 / 233)$ \\
\hline
\end{tabular}

Table 2. TM-scores and RMSD values for TM-alignments of core and cap regions of selected protein structures with those of hMGL (PDB code 3PE6). Results are ranked according to TM-score in the cap region. Results for the known MGLs, bMGL and YJU3p, in comparison with hMGL are separated.

\subsection{Activity assays reveal high activity of LipS towards MG substrates}

In order to test our hypothesis that enzymes with a cap architecture as observed in hMGL, Yju3p and bMGL exert activity against MGs, we measured enzymatic activities towards different substrates. To explore the substrate range we included pNP-acyl esters, MGs of different chain lengths and dioleoylglycerol in our assays. Since the selected candidates were all of bacterial origin, we used bMGL (Bacillus sp. H257) as reference MGL. Our in vitro experiments confirmed esterase activities for LipS, TtEst and CesA on short chain pNP esters as reported in the literature [26-28] and demonstrated esterase activity of bMGL on pNP esters (Fig. 3A and 3B). Enzymatic activities for Vc1974 and AV_0199 had not yet been reported. Our assays revealed that Avi_0199 has modest specific esterase activity towards $p N P-C 2\left(2.67 \pm 0.12 \mu \mathrm{mol} \cdot \mathrm{min}^{-1} \cdot \mathrm{mg}^{-1}\right)$ and $p \mathrm{NP}-\mathrm{C} 4\left(1.84 \pm 0.14 \mu \mathrm{mol} \cdot \mathrm{min}^{-1} \cdot \mathrm{mg}^{-1}\right)$. For Vc1974 esterase activity towards $p N P-C 2$, with a specific activity of $30.7 \cdot 10^{-3} \pm 4.1 \cdot 10^{-3} \mu \mathrm{mol} \cdot \mathrm{min}^{-1} \cdot \mathrm{mg}^{-1}$ and $p \mathrm{NP}$ C4, with a specific activity of $32.5 \cdot 10^{-3} \pm 3.2 \cdot 10^{-3} \mu \mathrm{mol} \cdot \mathrm{min}^{-1} \cdot \mathrm{mg}^{-1}$ was observed. LipS and Avi_0199 also hydrolyze $p$ NP substrates with longer chain lengths such as C8:0 and C10:0. In agreement with the literature, LipS is active against $p$ NP-C12:0 [27] (see Fig. 3A). LipS is the only enzyme that showed esterase activity at a level comparable, and even slightly higher than bMGL. In agreement with our hypothesis, LipS displayed high specific activity for MGs with different chain lengths as substrates. These experiments clearly identified LipS as a highly productive MGL (Fig. 3C): The highest MG hydrolase activity was measured for LipS with MG-C12:0 as the substrate at $104.8 \pm 2.2 \mu \mathrm{mol} \cdot \mathrm{min}^{-1} \cdot \mathrm{mg}^{-}$ ${ }^{1}$. This value is comparable to bMGL under the conditions tested, which shows a specific activity of $102.3 \pm 4.1 \mu \mathrm{mol} \cdot \mathrm{min}^{-1} \cdot \mathrm{mg}^{-1}$. The activities of the other enzymes against MG substrates were almost an 
order of magnitude lower. Avi_0199 exhibited activity against MG-C14:0 of 0.51 $\pm 0.01 \mu \mathrm{mol} \cdot \mathrm{min}^{-1} \cdot \mathrm{mg}^{-}$ ${ }^{1}$ (Fig. 3D). Vc1974 was also able to convert MGs but displayed low specific activity using MG-C12:0 as a substrate $\left(0.20 \pm 0.01 \mu \mathrm{mol} \cdot \mathrm{min}^{-1} \cdot \mathrm{mg}^{-1}\right)$. For CesA, little hydrolytic activity was detected with the highest activity measured with MG-C12:0 as the substrate $\left(0.11 \pm 0.03 \mu \mathrm{mol} \cdot \mathrm{min}^{-1} \cdot \mathrm{mg}^{-1}\right)$. For TtEst we observed very low activity (Fig. 3D). We also tested LipS, bMGL and Avi_0199, which displayed the highest MG hydrolase activities, for DG hydrolase activity, however no activity was detected against DG substrates (Fig. 3E).

A

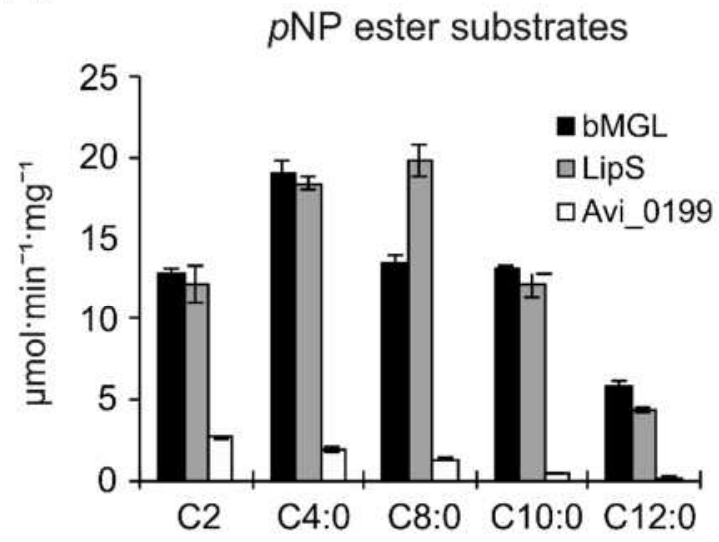

C

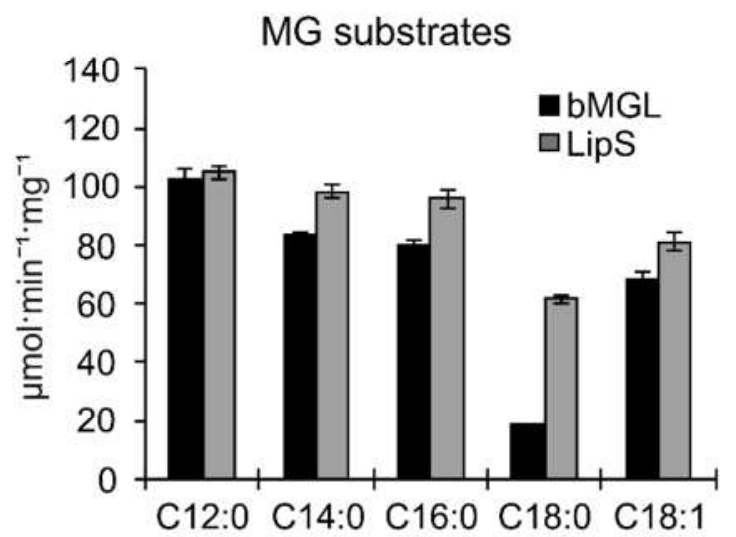

$\mathrm{E}$

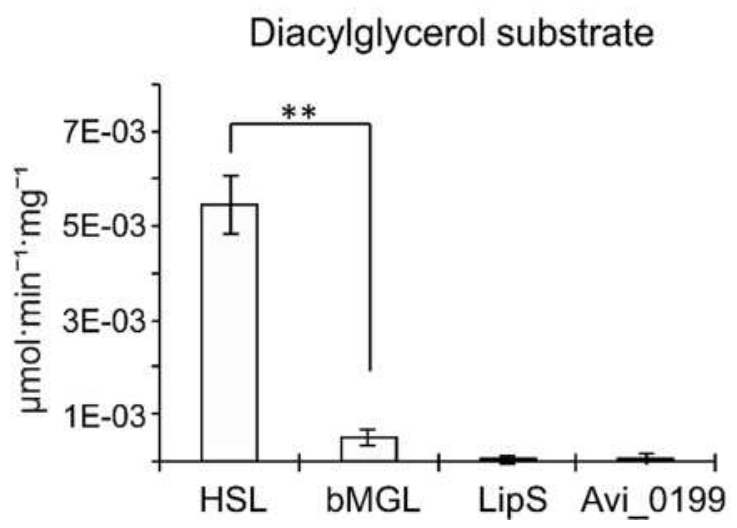

B

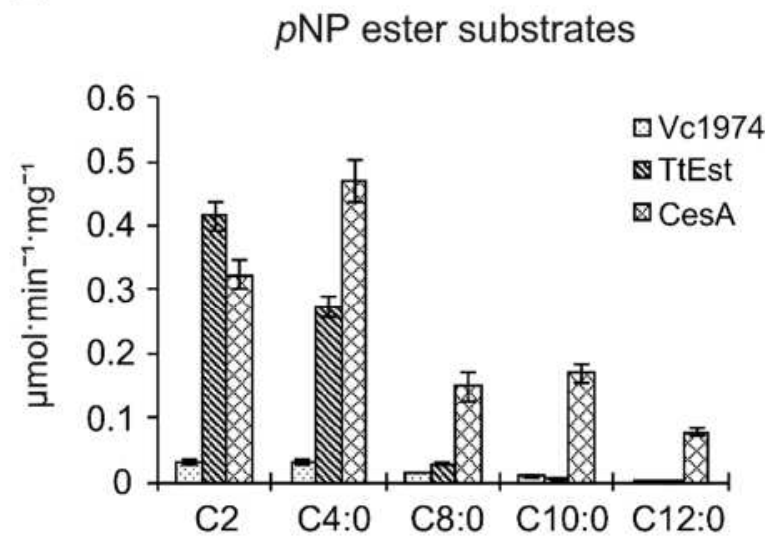

D

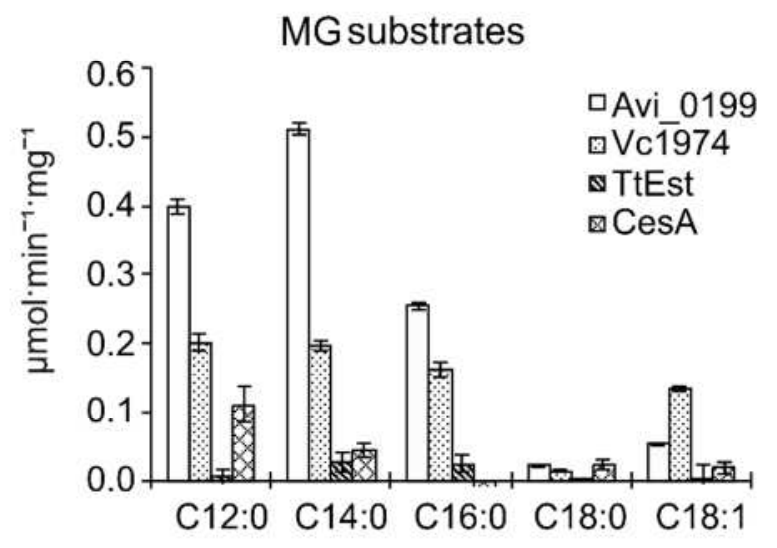

Figure 3. Comparison of specific activities of bMGL and candidate proteins towards a range of substrates. Data are presented as means \pm S.D. Statistical significance was determined by the Student's unpaired $t$ test (two- 
tailed). Group differences were considered statistically significant for $p<0.05(*), p<0.01(* *)$, and $p<0.001$ $(* * *)$. A, B) Hydrolysis of $p$-NP esters with different acyl chain lengths. C, D) Hydrolysis of MGs with different acyl chain length. E) Hydrolysis of dioleoylglycerol; HSL from mouse, expressed in Cos7 cells, is used as positive control.

We then determined the kinetic parameters of bMGL and LipS (Fig. 4). Time-course experiments were performed to ensure that measurements at 15 min incubation time are within the linear range of the reaction even at the highest substrate concentration used (Fig. 4A). As summarized in Table 3, bMGL and LipS showed similar values for $\mathrm{V}_{\max }, \mathrm{K}_{\mathrm{M}}$ and turnover rates.

\begin{tabular}{lccc} 
& $\mathrm{V}_{\max }\left[\mu \mathrm{M} \cdot \mathrm{min}^{-1}\right]$ & $\mathrm{K}_{\mathrm{M}}[\mu \mathrm{M}]$ & $\mathrm{k}_{\text {cat }}\left[\mathrm{min}^{-1}\right]$ \\
\hline bMGL & $16.5 \pm 0.6$ & $196.5 \pm 25.6$ & $5083 \pm 113$ \\
LipS & $18.2 \pm 0.6$ & $201.3 \pm 22.1$ & $5599 \pm 104$
\end{tabular}

Table 3. Kinetic parameters of bMGL and LipS.

A

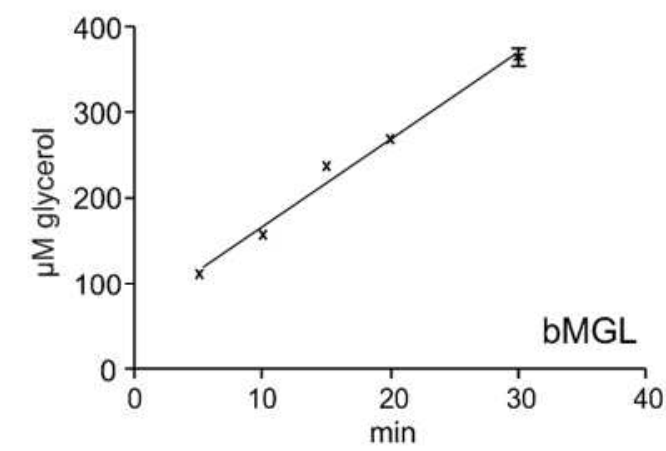

C

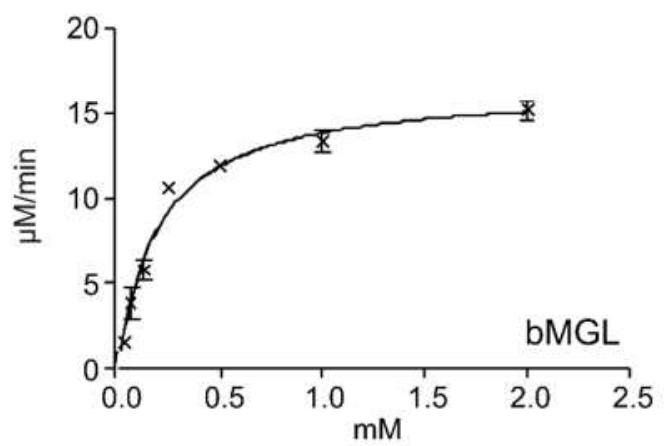

$\mathrm{B}$

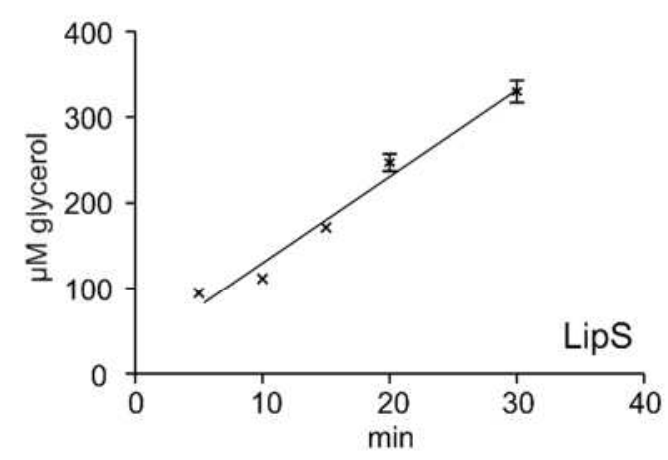

$\mathrm{D}$

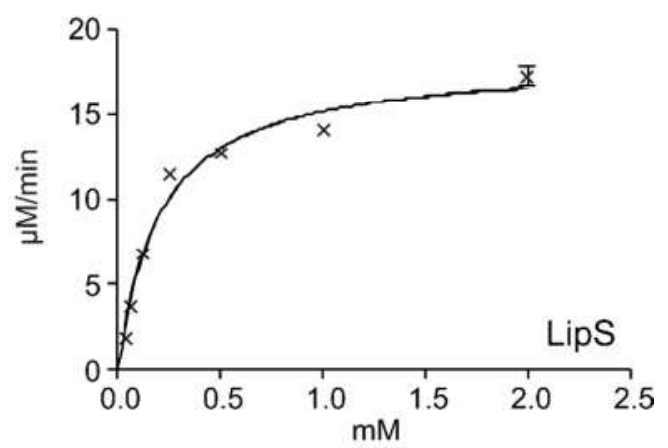

Figure 4. Kinetic characterization of the MG-hydrolase activities of LipS and bMGL. A,B) Time dependence with $3 \mathrm{nM}$ purified protein and $2 \mathrm{mM} \mathrm{MG-C12}$ shows a linear range up to $30 \mathrm{~min}$ for bMGL and LipS. C,D) Saturation kinetic studies on bMGL and LipS performed for $15 \mathrm{~min}$ with substrate MG-C12:0 and $3 \mathrm{nM}$ protein. $\mathrm{V}_{\max }$ and $\mathrm{K}_{\mathrm{M}}$ were determined by non-linear regression analysis. All assays were performed in triplicates, data are representative of at least three independent experiments. Data are presented as means \pm S.D. 


\section{Discussion}

The subclass of lipases with MG activity as studied here are ubiquitously found yet share low sequence identity (Table 1). Therefore, it is difficult to identify MGLs in different organisms solely based on the amino acid sequence. MGLS all share the ABH fold in the catalytic core domain and it is now straightforward to recognize members of the $A B H$ fold family based on their sequence. However, the enzymatic reactions catalyzed by members of this fold family are extremely diverse [25]. Hence, the classification of members of the ABH fold family according to their enzymatic activity poses a big challenge.

Lipases work at the interface between lipid and water and are often equipped with flexible cap domains of amphipathic nature. The hydrophobic nature of the cap can contribute to substrate recruitment and provides the hydrophobic surroundings important for substrate binding [35]. Several known lipases adopt an ABH fold and harbor a cap, but topology, size and shape differ extensively from the MGL subclass discussed here. For example, the cap insertion of the human gastric lipase (EC 3.1.1.3) spans residues Thr184-Asn308 and comprises 8 helices and several loop regions (PDB code 1 HLG [36]). However, only a region of 30 residues (His215-Cys244) is suggested to form the lid that opens for substrate access to the active site. In humans, hormone-sensitive lipase (EC 3.1.1.79) plays an important role in intracellular lipolysis. While an experimental structure is lacking, it is predicted that the $\mathrm{C}$-terminal catalytic domain adopts an $\mathrm{ABH}$ fold with a large insertion (residue Gly521-Tyr669) after strand 6 that forms the cap [37]. For another well studied lipase, CalB (EC 3.1.1.3) from Candida antarctica, the existence of a defined cap region is under debate (PDB code 5A71 [38]). We analyzed structures of 'classical' MGLs from human, yeast and a bacterial representative and developed a set of criteria to identify novel enzymes with MGL activity that is predominantly based on structural criteria of the cap region. It should be noted, that this search is not exhaustive for lipases with activity towards MGs. This approach will not pick up enzymes with different fold or cap regions, even though they might be able to hydrolyze MGs (as is the case for pancreatic lipase related protein $2[13,15]$ (EC 3.1.1.26, EC 3.1.1.3) or bile salt stimulated lipase [14] (EC 3.1.1.13, EC 3.1.1.3). We identified five candidate proteins that fulfilled our initial criteria - an $\mathrm{ABH}$ fold core and a cap insertion after strand $\beta 6$ forming a conserved shape over the binding pocket which harbors an intact catalytic triad. Among these, the thermostable protein LipS, showed the highest similarity to bMGL, hMGL and YJU3p. Notably, LipS and bMGL share $52.6 \%$ sequence identity overall and $52.2 \%$ in the core ( $40.9 \%$ in the cap region). LipS is identical to a lipase from Symbiobacterium thermophilum. These syntrophic bacteria are dependent on co-culture with a Bacillus strain [39] and it is suggested that around $25 \%$ of the protein coding genes are acquired by horizontal gene transfer [40]. One could speculate that LipS in Symbiobacterium thermophilum is the result of horizontal gene transfer from a Bacillus donor. This could explain the 
remarkable similarity to bMGL despite its phylogenetic distance. The structures exhibit striking similarities with an overall C $\alpha$ RMSD of $0.80 \AA$ over 234 residues. Compared to hMGL, the cap region of LipS displayed the highest TM-score of 0.430 amongst the candidate proteins. Next in the ranking below the LipS cap, the Vc1974 cap had a TM-score of 0.374. The TM-score as well as RMSD value for the Vc1974 cap are both significantly lower compared to LipS, thus highlighting that the LipS cap architecture shows by far the highest similarity to hMGL. The other candidates displayed lower TM-scores in the cap, even though the core scores were comparable to those of LipS. We then proved that LipS indeed displays MGL activity as we predicted based on our analysis. Under our experimental conditions, LipS even exhibited higher specific activity than bMGL. The substrate preference is comparable to bMGL; the highest activity was observed against MG-C12. Thus, TM scores can be used to qualitatively predict MGL activity, yet cannot be interpreted as direct quantifier of MGL activity. In kinetic studies we showed that the enzymatic properties of LipS and bMGL are similar, with comparable values obtained for the maximal velocity, turnover number and $\mathrm{K}_{\mathrm{M}}$ for the substrate MG-C12 $\left(\mathrm{K}_{\mathrm{M}} \sim 200 \mu \mathrm{M}\right)$. This resemblance can be explained by the similar amino acid composition in the binding pockets. Typically, the substrate binding pocket of any MGL is formed by residues from the cap and core. In LipS, 15 hydrophobic residues, six in the cap and nine in the core (Phe58, Leu125, Met127, Ala 152, Met155, Leu160, Leu163, Leu173, Ile176, Ala 189, Ile198, Leu201, Val229, Val230, Val258) form the pocket, whereas in bMGL 13 equivalent residues - six locate to the cap and seven to the core (Phe29, Val 98, Ala122, Ile125, Ile128, Met132, Leu138, Ile142, Ile145, Leu167, Leu170, Met174 and Val198)- are present. Based on studies with hMGL, bMGL and YJU3p, typical enzymatic MGL characteristics are a specific activity in the high $\mu \mathrm{mol} \cdot \mathrm{min}^{-1} \cdot \mathrm{mg}^{-1}$ range, $\mathrm{K}_{\mathrm{M}}$ values in the $\mu \mathrm{M}$ range and substrate preference towards MGs, and only very low or no activity towards DG or TG. All these criteria are clearly met by LipS and we therefore identified LipS as a novel thermophilic MGL.

\section{Conclusion}

We identified LipS as a novel MGL based on structural criteria beyond the overall fold. We showed that high TM-scores and low RMSDs of cap alignments to hMGL can be taken into consideration for the identification of enzymes with MGL activity. These are a more reliable indicator than the corresponding values for the core $\mathrm{ABH}$ domains alone. We also identified enzymes with low level MGL activity (Avi_0199 and Vc1974) using these criteria. Together, these data support our hypothesis that the conserved cap architecture can be utilized as criterion to identify lipases with MGL activity.

\section{Acknowledgements}

This work was supported by the Doctoral School "DK Molecular Enzymology" Grant W09 and by 
Project P24857 both granted by Austrian Science Fund (FWF). We thank Jennifer Chow and Wolfgang

Streit from the University of Hamburg for providing the LipS plasmid.

\section{Author contributions}

Conceived and designed the experiments: AL LRB PA MO. Performed the experiments: AL LRB.

Analyzed the data: AL LRB ID MO. Wrote the paper: LRB PA ID MO.

\section{Literature}

[1] T.P. Dinh, D. Carpenter, F.M. Leslie, T.F. Freund, I. Katona, S.L. Sensi, S. Kathuria, D. Piomelli, Brain monoglyceride lipase participating in endocannabinoid inactivation, Proc. Natl. Acad. Sci. 99 (2002) 10819-10824. doi:10.1073/pnas.152334899.

[2] K. Côtes, R. Dhouib, I. Douchet, H. Chahinian, A. de Caro, F. Carrière, S. Canaan, Characterization of an exported monoglyceride lipase from Mycobacterium tuberculosis possibly involved in the metabolism of host cell membrane lipids, Biochem. J. 408 (2007) 417427. doi:10.1042/BJ20070745.

[3] U. Taschler, F.P.W. Radner, C. Heier, R. Schreiber, M. Schweiger, G. Schoiswohl, K. Preiss-Landl, D. Jaeger, B. Reiter, H.C. Koefeler, J. Wojciechowski, C. Theussl, J.M. Penninger, A. Lass, G. Haemmerle, R. Zechner, R. Zimmermann, Monoglyceride Lipase Deficiency in Mice Impairs Lipolysis and Attenuates Diet-induced Insulin Resistance, J. Biol. Chem. 286 (2011) 1746717477. doi:10.1074/jbc.M110.215434.

[4] P. Nilsson-Ehle, P. Belfrage, A monoglyceride hydrolyzing enzyme in human postheparin plasma, Biochim. Biophys. Acta. 270 (1972) 60-64.

[5] J.R. Senior, K.J. Isselbacher, Demonstration of an intestinal monoglyceride lipase: an enzyme with a possible role in the intracellular completion of fat digestion., J. Clin. Invest. 42 (1963) 187-195. doi:10.1172/JCl104705.

[6] L. Scalvini, F. Vacondio, M. Bassi, D. Pala, A. Lodola, S. Rivara, K.-M. Jung, D. Piomelli, M. Mor, Free-energy studies reveal a possible mechanism for oxidation-dependent inhibition of MGL, Sci. Rep. 6 (2016). doi:10.1038/srep31046.

[7] H. Tornqvist, P. Belfrage, Purification and some properties of a monoacylglycerol hydrolyzing enzyme of rat adipose tissue, J. Biol. Chem. 251 (1976) 813-819.

[8] C. Heier, U. Taschler, S. Rengachari, M. Oberer, H. Wolinski, K. Natter, S.D. Kohlwein, R. Leber, R. Zimmermann, Identification of Yju3p as functional orthologue of mammalian monoglyceride lipase in the yeast Saccharomyces cerevisiae, Biochim. Biophys. Acta - Mol. Cell Biol. Lipids. 1801 (2010) 1063-1071.

[9] R.J. Kim, H.J. Kim, D. Shim, M.C. Suh, Molecular and biochemical characterizations of the monoacylglycerol lipase gene family of Arabidopsis thaliana, Plant J. 85 (2016) 758-771. doi:10.1111/tpj.13146.

[10] T. Sakiyama, T. Yoshimi, A. Miyake, M. Umeoka, A. Tanaka, S. Ozaki, K. Nakanishi, Purification and characterization of a monoacylglycerol lipase from Pseudomonas sp. LP7315, J. Biosci. Bioeng. 91 (2001) 27-32.

[11] S. Imamura, S. Kitaura, Purification and characterization of a monoacylglycerol lipase from the moderately thermophilic Bacillus sp. H-257, J. Biochem. (Tokyo). 127 (2000) 419-425.

[12] R. Dhouib, F. Laval, F. Carriere, M. Daffe, S. Canaan, A Monoacylglycerol Lipase from Mycobacterium smegmatis Involved in Bacterial Cell Interaction, J. Bacteriol. 192 (2010) 47764785. doi:10.1128/JB.00261-10. 
[13] C. Eydoux, S. Spinelli, T.L. Davis, J.R. Walker, A. Seitova, S. Dhe-Paganon, A. De Caro, C. Cambillau, F. Carrière, Structure of Human Pancreatic Lipase-Related Protein 2 with the Lid in an Open Conformation ${ }^{\dagger},{ }^{\ddagger}$, Biochemistry (Mosc.). 47 (2008) 9553-9564. doi:10.1021/bi8005576.

[14] C.S. Wang, A. Kuksis, F. Manganaro, J.J. Myher, D. Downs, H.B. Bass, Studies on the substrate specificity of purified human milk bile salt-activated lipase, J. Biol. Chem. 258 (1983) 91979202.

[15] K. Dridi, S. Amara, S. Bezzine, J.A. Rodriguez, F. Carrière, H. Gaussier, Partial deletion of beta9 loop in pancreatic lipase-related protein 2 reduces enzyme activity with a larger effect on long acyl chain substrates, Biochim. Biophys. Acta. 1831 (2013) 1293-1301.

[16] T. Bertrand, F. Augé, J. Houtmann, A. Rak, F. Vallée, V. Mikol, P.F. Berne, N. Michot, D. Cheuret, C. Hoornaert, M. Mathieu, Structural Basis for Human Monoglyceride Lipase Inhibition, J. Mol. Biol. 396 (2010) 663-673.

[17] G. Griebel, P. Pichat, S. Beeské, T. Leroy, N. Redon, A. Jacquet, D. Françon, L. Bert, L. Even, M. Lopez-Grancha, T. Tolstykh, F. Sun, Q. Yu, S. Brittain, H. Arlt, T. He, B. Zhang, D. Wiederschain, T. Bertrand, J. Houtmann, A. Rak, F. Vallée, N. Michot, F. Augé, V. Menet, O.E. Bergis, P. George, P. Avenet, V. Mikol, M. Didier, J. Escoubet, Selective blockade of the hydrolysis of the endocannabinoid 2-arachidonoylglycerol impairs learning and memory performance while producing antinociceptive activity in rodents, Sci. Rep. 5 (2015). doi:10.1038/srep07642.

[18] G. Labar, C. Bauvois, F. Borel, J.-L. Ferrer, J. Wouters, D.M. Lambert, Crystal structure of the human monoacylglycerol lipase, a key actor in endocannabinoid signaling, ChemBioChem. 11 (2010) 218-227.

[19] C. Schalk-Hihi, C. Schubert, R. Alexander, S. Bayoumy, J.C. Clemente, I. Deckman, R.L. DesJarlais, K.C. Dzordzorme, C.M. Flores, B. Grasberger, J.K. Kranz, F. Lewandowski, L. Liu, H. Ma, D. Maguire, M.J. Macielag, M.E. McDonnell, T. Mezzasalma Haarlander, R. Miller, C. Milligan, C. Reynolds, L.C. Kuo, Crystal structure of a soluble form of human monoglyceride lipase in complex with an inhibitor at 1.35 A resolution: Crystal Structure of Monoglyceride Lipase, Protein Sci. 20 (2011) 670-683. doi:10.1002/pro.596.

[20] S. Rengachari, G.A. Bezerra, L. Riegler-Berket, C.C. Gruber, C. Sturm, U. Taschler, A. Boeszoermenyi, I. Dreveny, R. Zimmermann, K. Gruber, M. Oberer, The structure of monoacylglycerol lipase from Bacillus sp. $\mathrm{H} 257$ reveals unexpected conservation of the cap architecture between bacterial and human enzymes, Biochim. Biophys. Acta BBA - Mol. Cell Biol. Lipids. 1821 (2012) 1012-1021. doi:10.1016/j.bbalip.2012.04.006.

[21] S. Rengachari, P. Aschauer, M. Schittmayer, N. Mayer, K. Gruber, R. Breinbauer, R. BirnerGruenberger, I. Dreveny, M. Oberer, Conformational Plasticity and Ligand Binding of Bacterial Monoacylglycerol Lipase, J. Biol. Chem. 288 (2013) 31093-31104. doi:10.1074/jbc.M113.491415.

[22] P. Aschauer, S. Rengachari, J. Lichtenegger, M. Schittmayer, K.M.P. Das, N. Mayer, R. Breinbauer, R. Birner-Gruenberger, C.C. Gruber, R. Zimmermann, K. Gruber, M. Oberer, Crystal structure of the Saccharomyces cerevisiae monoglyceride lipase Yju3p, Biochim. Biophys. Acta BBA - Mol. Cell Biol. Lipids. 1861 (2016) 462-470. doi:10.1016/j.bbalip.2016.02.005.

[23] D.L. Ollis, E. Cheah, M. Cygler, B. Dijkstra, F. Frolow, S.M. Franken, M. Harel, S.J. Remington, I. Silman, J. Schrag, J.L. Sussman, K.H.G. Verschueren, A. Goldman, The $\alpha$ / 8 hydrolase fold, Protein Eng. Des. Sel. 5 (1992) 197-211. doi:10.1093/protein/5.3.197.

[24] M. Nardini, B.W. Dijkstra, Alpha/beta hydrolase fold enzymes: the family keeps growing, Curr. Opin. Struct. Biol. 9 (1999) 732-737.

[25] M. Holmquist, Alpha/Beta-hydrolase fold enzymes: structures, functions and mechanisms, Curr. Protein Pept. Sci. 1 (2000) 209-235.

[26] M.J. Dröge, R. Bos, Y.L. Boersma, W.J. Quax, Comparison and functional characterisation of three homologous intracellular carboxylesterases of Bacillus subtilis, J. Mol. Catal. B Enzym. 32 (2005) 261-270. doi:10.1016/j.molcatb.2004.12.010. 
[27] J. Chow, F. Kovacic, Y. Dall Antonia, U. Krauss, F. Fersini, C. Schmeisser, B. Lauinger, P. Bongen, J. Pietruszka, M. Schmidt, I. Menyes, U.T. Bornscheuer, M. Eckstein, O. Thum, A. Liese, J. Mueller-Dieckmann, K.-E. Jaeger, W.R. Streit, The Metagenome-Derived Enzymes LipS and LipT Increase the Diversity of Known Lipases, PLoS ONE. 7 (2012) e47665. doi:10.1371/journal.pone.0047665.

[28] C. Sayer, M.N. Isupov, E. Bonch-Osmolovskaya, J.A. Littlechild, Structural studies of a thermophilic esterase from a new Planctomycetes species, Thermogutta terrifontis, FEBS J. 282 (2015) 2846-2857. doi:10.1111/febs.13326.

[29] E. Krissinel, K. Henrick, Secondary-structure matching (SSM), a new tool for fast protein structure alignment in three dimensions, Acta Crystallogr. Sect. D. 60 (2004) 2256-2268. doi:10.1107/S0907444904026460.

[30] Schrödinger, LLC, The PyMOL Molecular Graphics System, Version 1.8, (2015).

[31] Y. Zhang, TM-align: a protein structure alignment algorithm based on the TM-score, Nucleic Acids Res. 33 (2005) 2302-2309. doi:10.1093/nar/gki524.

[32] S. Tan, R.C. Kern, W. Selleck, The PST44 polycistronic expression system for producing protein complexes in Escherichia coli, Protein Expr. Purif. 40 (2005) 385-395. doi:10.1016/j.pep.2004.12.002.

[33] J. Pei, B.-H. Kim, N.V. Grishin, PROMALS3D: a tool for multiple protein sequence and structure alignments, Nucleic Acids Res. 36 (2008) 2295-2300. doi:10.1093/nar/gkn072.

[34] H.J. Rozeboom, L.F. Godinho, M. Nardini, W.J. Quax, B.W. Dijkstra, Crystal structures of two Bacillus carboxylesterases with different enantioselectivities, Biochim. Biophys. Acta BBA Proteins Proteomics. 1844 (2014) 567-575. doi:10.1016/j.bbapap.2014.01.003.

[35] F.I. Khan, D. Lan, R. Durrani, W. Huan, Z. Zhao, Y. Wang, The Lid Domain in Lipases: Structural and Functional Determinant of Enzymatic Properties, Front. Bioeng. Biotechnol. 5 (2017). doi:10.3389/fbioe.2017.00016.

[36] A. Roussel, S. Canaan, M.-P. Egloff, M. Riviere, L. Dupuis, R. Verger, C. Cambillau, Crystal Structure of Human Gastric Lipase and Model of Lysosomal Acid Lipase, Two Lipolytic Enzymes of Medical Interest, J. Biol. Chem. 274 (1999) 16995-17002. doi:10.1074/jbc.274.24.16995.

[37] S.J. Yeaman, Hormone-sensitive lipase - new roles for an old enzyme, Biochem. J. 379 (2004) 11-22. doi:10.1042/bj20031811.

[38] B. Stauch, S.J. Fisher, M. Cianci, Open and closed states of Candida antarctica lipase B: protonation and the mechanism of interfacial activation, J. Lipid Res. 56 (2015) 2348-2358. doi:10.1194/jlr.M063388.

[39] K. Ueda, Genome sequence of Symbiobacterium thermophilum, an uncultivable bacterium that depends on microbial commensalism, Nucleic Acids Res. 32 (2004) 4937-4944. doi:10.1093/nar/gkh830.

[40] H. Nishida, C.-S. Yun, Phylogenetic and Guanine-Cytosine Content Analysis of Symbiobacterium thermophilum Genes, Int. J. Evol. Biol. 2011 (2011) 1-5. doi:10.4061/2011/634505. 